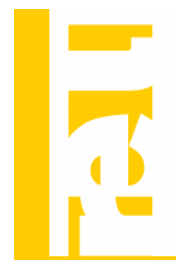

\title{
Disparidades entre educación formal y educación en el puesto de trabajo
}

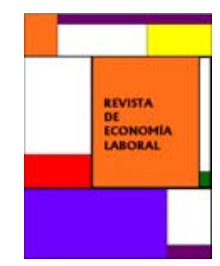

\author{
Maite Blázquez*,1, José Ramos ${ }^{\dagger}$ \\ *Universidad Autónoma de Madrid, †Universidad Europea de Madrid
}

Recibido el 12 de septiembre de 2007; aceptado el 23 de abril de 2008

\section{Resumen}

El presente trabajo pretende desagregar el capital humano en sus dos componentes principales: la educación formal en la escuela y la formación en el trabajo. Analizando el comportamiento de ambas formas de acumulación de capital humano, se pretende conseguir un mejor conocimiento del comportamiento de los salarios en la economía española. La investigación viene motivada por el incremento sustancial del nivel educativo de la fuerza laboral. El porcentaje de población activa con educación superior ha aumentado considerablemente, lo cual ha llevado a plantearnos la necesidad de evaluar la coordinación entre las distintas formas de acumulación de capital humano.

Palabras Clave: Disparidades salariales, educación formal, formación en el puesto de trabajo.

Clasificación JEL: I20, J21, J24, J30, J31.

\begin{abstract}
This paper is aimed at decomposing human capital in its two main components: schooling and on-the-job training. Analyzing both forms of human capital accumulation we aim at getting a better understanding of wage determination in the Spanish economy. The motivation of the paper comes from the remarkably increase in the educational attainments of the workforce in the last decades. The share of labour force with higher education has experienced an upwards trend, what has lead to the necessity of evaluating the role of both types of human capital accumulation in the economic performance.
\end{abstract}

Key words: Wage disparities, educational level, on-the-job training JEL Classification: I20, J21, J24, J30, J31.

1 Autora para correspondencia: maite.blazquez@uam.es. Agradecemos a los participantes de las VII Jornadas de Economía Laboral celebradas en Gran Canaria en julio de 2007, a los participantes de las XVI Jornadas de la Asociación de Economía de la Educación, así como a los participantes del X Encuentro de Economía Aplicada sus comentarios y sugerencias.

(C) Revista de Economía Laboral 


\section{Introducción}

En las últimas décadas ha aumentado el interés por el crecimiento económico y en particular por comprender mejor los patrones de crecimiento de los países. Este trabajo tiene como punto de partida la literatura de crecimiento endógeno desarrollada entre otros por Lucas (1988), Romer (1986) y Rebelo (1991), y que señalan el capital humano como la herramienta clave para entender los procesos de crecimiento de las economías. El objetivo central es realizar una pequeña aportación al entendimiento de lo que se conoce como Desarrollo Económico de las Naciones.

El presente trabajo pretende desagregar el capital humano en sus dos componentes principales: la educación formal en la escuela y la formación en el trabajo. Analizando el comportamiento de ambas formas de acumulación de capital humano por separado, se pretende conseguir un mejor conocimiento de los distintos procesos de crecimiento y desarrollo que se observan dentro de los países más avanzados.

El trabajo viene motivado por algunos hechos empíricos que se han puesto de manifiesto en los últimos años. Por un lado, la existencia de grandes disparidades en los niveles de renta entre países, los cuales no sólo no han convergido, sino que han sufrido un proceso de mayor divergencia. $\mathrm{Y}$ en segundo lugar el incremento sustancial, especialmente en los países desarrollados, del nivel educativo de la fuerza laboral, de manera que el porcentaje de población activa con niveles de educación superior ha aumentado considerablemente en dichos países.

Estas cuestiones llevan a plantearse la necesidad de evaluar la coordinación entre las distintas formas de acumulación de capital humano, ya que entendiendo su distinto comportamiento podemos llegar a entender mejor las diferencias en los niveles de renta. El hecho de considerar dos tipos de capital humano con características distintas, permitirá analizar bajo que circunstancias es mejor una forma de capital humano $u$ otra, en aras de mejorar los niveles de productividad y de renta de la economía.

Este trabajo se estructura en dos partes diferenciadas, que convergen a un objetivo único: entender la relación entre las distintas formas de capital humano y como cada una de ellas determina la productividad de los individuos, y por ende, el nivel renta per capita de los países.

En primer lugar se analizará un modelo de crecimiento endógeno, donde la fuente de endogeneidad es doble, por un lado la 
educación formal que reciben los trabajadores y por otro lado la cualificación adquirida mediante formación en su puesto de trabajo. Partiendo de que existen dos formas de acumular capital humano, el modelo pretende explicar la importancia relativa de ambos elementos en las diferencias de productividad y de renta observadas en los países desarrollados.

En este modelo se asumirá que los agentes deciden su nivel de educación y que las empresas completan esta educación con la formación necesaria para el aprovechamiento eficiente de la tecnología disponible. El objetivo consiste en determinar la proporción óptima entre estas dos formas de acumulación de capital humano. Por un lado, como señala Lucas (1993), la inversión en educación formal es una decisión de los individuos de cómo asignar el tiempo disponible. El nivel de educación adquirido permite a los trabajadores adquirir un capital humano no-específico que va a constituir un factor determinante de su nivel de productividad. Por otro lado las empresas necesitan formar a sus trabajadores para proporcionarles aquellas cualificaciones, de carácter específico, necesarias para aprovechar de la manera más eficiente el nivel tecnológico disponible.

En esta primera parte del trabajo, se utilizarán datos de la economía española para analizar el peso que tienen ambos tipos de capital humano a la hora de explicar la producción de los distintos sectores de actividad de nuestra economía. Partiendo de una función de producción tipo $C E S$, se analizará el grado de complementariedad entre ambas formas de capital humano, así como de éstas con el stock de capital físico.

La segunda parte del presente trabajo se centra en un análisis micro, donde se utilizarán datos de la Encuesta del Panel de Hogares de la Unión Europea (PHOGUE 1995-2001) para analizar el impacto que tiene la educación recibida por los trabajadores tanto en la escuela, como en su puesto de trabajo, sobre los salarios.

El trabajo se estructura de la siguiente manera. En la Sección 2 se revisarán los trabajos más destacados de la literatura relacionados con el capital humano y su impacto tanto en el crecimiento económico, como en la renta de los individuos. En la Sección 3 se desarrollará un modelo básico de crecimiento endógeno, el cual servirá como referente en la Sección 4 donde se analizará el comportamiento de las distintas formas de capital humano por sectores de actividad. En la Sección 5 se estudiará el papel que juegan ambas formas de capital humano sobre el nivel de renta de los trabajadores utilizando los datos del PHOGUE. Por último en la Sección 6 se extraerán las principales conclusiones del trabajo. 


\section{Literatura Previa}

La literatura que ha servido como referencia en este trabajo puede agruparse en dos grandes bloques. Por un lado aquellos trabajos que han analizado la influencia del capital humano sobre el crecimiento económico y las diferencias de renta entre países, ya sea desde una perspectiva teórica o empírica. $\mathrm{Y}$ en segundo lugar los trabajos relacionados con el mercado de trabajo, que han estudiado el papel de la educación formal y la formación en el puesto de trabajo en la determinación de las ganancias salariales de los trabajadores.

En cuanto al primer bloque de trabajos, es importante señalar que no sólo se han centrado en explicar los patrones de crecimiento económico y las desigualdades de renta de los distintos países, así como el papel que ha jugado el capital humano, sino también en cómo estas disparidades han persistido en el tiempo.

Entre los trabajos pioneros dentro de este primer bloque destacan los de Schultz $(1960,1961)$ que ofrecen las primeras estimaciones de la contribución del capital humano al crecimiento económico. Posteriormente, el trabajo de Denison (1962) proporcionó evidencia empírica que incentivó a los teóricos a incorporar el capital humano en los modelos formales de crecimiento económico. Así, en el trabajo de Uzawa (1965) se presenta una ampliación de los trabajos de Cass (1965) y Koopmans (1965), incorporando el sector educativo dentro del modelo de crecimiento económico. En la misma línea, en el trabajo de Romer (1986), que supuso una importante contribución a esta literatura, se presenta un modelo de crecimiento a largo plazo donde el capital humano se considera como un factor productivo en la función de producción, que conduce a un aumento en la productividad marginal.

Trabajos posteriores como el de Lucas (1988) señalan al capital humano como factor clave que permite explicar el crecimiento, así como las grandes disparidades de renta entre los distintos países. En esta línea, los trabajos de Tamura (1991) y O'neill (1995) ponen de manifiesto que la convergencia en niveles de capital humano de las economías es un factor muy importante para conseguir la convergencia real en términos de renta, hasta el punto de que esta última no sería posible si no existiese la primera.

A raíz de estos primeros trabajos, y especialmente del trabajo de Lucas (1988), surge una de las contribuciones más destacadas en esta literatura, el trabajo de Prescott (1997), donde se vuelve a resaltar una vez más las grandes diferencias en productividad, y por tanto en niveles de renta, entre los distintos países. Tal y como señala el autor, y en 
línea con trabajos anteriores, la teoría neoclásica no sirve para explicar las diferencias de rentas entre países. Prescott plantea que estas diferencias se deben fundamentalmente a diferencias en la habilidad de los trabajadores ante cambios en las prácticas de trabajo. Además este autor señala la eficiencia en el uso de la tecnología existente como elemento clave de las diferencias en productividad anteriormente mencionadas.

Más recientemente, algunos trabajos han analizado la relación entre inversión en capital humano, crecimiento de la productividad y la existencia de ciclos de crecimiento endógenos, como el trabajo de Kaas y Stefan (2007).

Junto con estos trabajos teóricos surge una literatura que analiza el problema del crecimiento y de los niveles de renta per capita desde una perspectiva empírica. Entre estos trabajos se encuentran los de Mankiw et al. (1992), Parente y Prescott (1993), y MacGrattan y Schmitz (1998). En concreto, estos últimos han señalado que el producto interior bruto en Estados Unidos es 30 veces el de un país pobre como Etiopía, y que además los países que más crecen lo hacen a una tasa del 9\% anual, mientras que hace 100 años la tasa máxima era tan sólo del 2\%. La conclusión más relevante del trabajo de MacGrattan y Schmitz, en sintonía con el trabajo de Hall y Jones (1999), es que mientras existe convergencia en los niveles de renta entre los países industrializados hay una clara divergencia de rentas a nivel mundial. Otros trabajos como el de Temple, J. (2001) proporcionan evidencia empírica de los beneficios de la inversión educación sobre la productividad laboral y el crecimiento económico en los países de la OCDE.

Además del importante papel que ha jugado el capital humano a la hora de explicar los patrones de crecimiento económico y las disparidades de renta entre los países, cabe señalar que en las últimas décadas se ha producido un incremento importante del nivel educativo de la sociedad, especialmente en los países desarrollados, tal y como señalan algunos trabajos como los de Barro y Lee (1993) y Benhabib y Spiegel (1994). Así, en el trabajo de Barro y Lee (1993) se pone de manifiesto que mientras que las tasas de escolarización en educación primaria y secundaria eran muy similares en países en desarrollo y países desarrollados, las correspondientes al nivel de educación universitaria son significativamente mayores en estos últimos, llegando a duplicarse entre 1960 y 1985.

El trabajo de Helpman y Rangel (1998) apunta a que una de las explicaciones al importante aumento de los niveles educativos que se ha observado en los países desarrollados ha sido el alto grado de desarrollo tecnológico experimentado en los mismos, lo que ha provocado un 
aumento en la demanda de trabajadores con mayores niveles de cualificación general.

Un trabajo más reciente, en línea con los anteriores, y en sintonía con el presente trabajo es el de Serrano-Martínez (2003), el cual parte de la idea de que el crecimiento económico entraña un cambio en la estructura sectorial. En este trabajo se pone de manifiesto que el capital humano varía según el sector. Entre sus conclusiones principales destaca que la agricultura es la rama con un menor nivel de cualificación, seguida de la construcción. Por otro lado tanto en la agricultura como en el sector energético, el capital humano no tiene efecto, ni en tasa ni en nivel. Por el contrario, en el sector industrial el capital humano sí afecta de modo significativo al crecimiento industrial tanto en términos de niveles como tasas de crecimiento. Del trabajo se desprende que la contribución del capital humano al crecimiento se produce mediante su efecto directo sobre la capacidad productiva de la economía. Esta misma conclusión aparecía ya en trabajos previos como el de Alan (1993) y De la Fuente y Da Rocha (1996), donde se pone de manifiesto la necesidad de prestar mayor atención a la formación técnica y profesional, y aumentar los recursos para mejorar la calidad de la formación. Como señalan estos autores, en la medida en que la educación incrementa la capacidad de los individuos para procesar información y tomar decisiones, contribuye también a aumentar su productividad y su capacidad de aprendizaje. De estos y otros trabajos como el de Lynch (1993) se concluye que existe un cierto grado de complementariedad entre educación formal y formación en el puesto de trabajo.

Los trabajos anteriormente citados estudiaban el capital humano como elemento clave del crecimiento económico. Sin embargo, no conviene olvidar el segundo bloque de literatura que analiza la influencia del capital humano sobre el mercado laboral, y en particular aquellos trabajos que han estudiado el impacto de la educación y la formación en el puesto de trabajo sobre las ganancias salariales de los trabajadores. Los trabajos pioneros dentro de este bloque de la literatura son los de Becker (1962) y Mincer (1974), en los que se concluye que las ganancias salariales aumentan de manera significativa con el stock de capital humano.

A partir del trabajo de Mincer encontramos una serie de trabajos que estiman la ecuación de salarios Minceriana para examinar la influencia de la educación recibida en la escuela y la formación obtenida en el puesto de trabajo sobre las ganancias salariales. Esta literatura aparece revisada en los trabajos de Willis (1986), Card (1995), Psacharopoulos (1981, 1985) y Psacharopoulos y Patrinos (2002). 
Más recientemente, el trabajo de Brunello y Comi (2000) proporciona evidencia de los rendimientos positivos de la inversión en educación terciaria para 11 países europeos, encontrando diferencias en función del grado de corporativismo y de las características del sistema educativo de cada país. En la misma línea se encuentran los trabajos de Harmon et al. (2001) y De la Fuente y Jimeno (2005). Para un estudio a nivel europeo, Harmon et al. (2001) encuentran que un año adicional de educación aumenta los salarios individuales en un $6.5 \%$, y que dicha cifra puede llegar a un $9 \%$ cuando el análisis se limita a aquellos Estados Miembros con mercados laborales menos regulados. Por su parte, De la Fuente y Jimeno (2005) estiman unos rendimientos privados de la educación que oscilan entre el $7.5 \%$ y el $10 \%$ para la mayoría de los países europeos.

Algunos trabajos recientes han tratado de analizar conjuntamente los efectos de la inversión en educación formal tanto a nivel micro como a nivel macro. Así, los trabajos de De la Fuente y Doménech (2006) y Cohen y Soto (2001) ponen de manifiesto que la acumulación de capital humano, además de contribuir al crecimiento de los salarios de los trabajadores, resulta una importante fuente de externalidades positivas que contribuyen al crecimiento económico de un país.

Además de los trabajos que analizan los rendimientos de la educación formal, encontramos numerosos trabajos que se han centrado en analizar el efecto de la formación en el puesto de trabajo sobre las ganancias salariales (Duncan y Hoffman, 1981; Altonji y Spletzer, 1991; Barron, Black y Loewenstein, 1993; y Acemoglu y Pischke, 1999b).

Por último, cabe mencionar algunos trabajos que se han centrado en analizar el grado de complementariedad entre la educación formal y la formación en el puesto de trabajo, como el de Brunello (2001) donde se lleva a cabo un análisis para 13 países europeos utilizando la Encuesta de Panel de Hogares de la Unión Europea. El principal resultado de este trabajo es que los individuos con mayores niveles de educación formal tienden a invertir más en formación en el puesto de trabajo, si bien se observan algunas diferencias importantes entre países en base a las características del sistema educativo y de las instituciones del mercado de trabajo.

\section{Modelo}

Se parte de un modelo de crecimiento endógeno similar al desarrollado por Lucas (1993) y Prescott (1997) con economía cerrada, 
sin sector público y sin externalidades. Para simplificar se asume que la población tiene una tasa de crecimiento natural nula $(n=0)$.

Se trata de un modelo de Equilibrio General con dos agentes: hogares y empresas. En la economía se produce un único bien que se puede destinar al consumo o a la inversión. Por otro lado, existen tres sectores de actividad. Uno dedicado a la producción del único bien final; otro dedicado a la producción de educación formal en la escuela; y el tercero dedicado a la producción de formación dentro de la empresa. Se asume que tanto la educación como la formación poseen las mismas características y que se deprecian a la misma tasa $\delta$.

Por un lado el individuo decide cuanto tiempo dedica a educarse, $n_{s}$, cuanto tiempo destina a trabajar, $n_{f}$, y cuanto a ocio, $n_{l}$. Y una vez conocido $n_{f}$ la empresa decide cuanto tiempo usa para formar al individuo, $n_{t}$, y cuanto destina a producir, $n_{p}$.

\subsection{Hogares}

\subsubsection{Utilidad}

El individuo vive durante infinitos periodos $(T=\infty)$. Este individuo representativo puede considerarse como un hogar que toma la decisión en un horizonte infinito, donde $\rho$ es la tasa de preferencia por el presente.

El nivel de utilidad del hogar en el momento $t$ depende del nivel de consumo $\left(C_{t}\right)$ y de su nivel de Ocio $\left(L_{t}\right): 2$

$$
U\left(C_{t}, L_{t}\right)=\ln \left(C_{t}\right)+\gamma \ln \left(L_{t}\right)
$$

donde $U_{1}>0, U_{2}>0$ y $U_{i i}<0$ para $\forall i=1,2$. Se asume $N$ individuos idénticos, y que la masa total de individuos en todo momento $t$ es igual

2 De acuerdo con Sala-i-Martin (1994, página 45), utilizamos una función de utilidad de la forma:

$$
U\left(C_{t}, L_{t}\right)=\frac{\left[C_{t} L_{t}^{\gamma}\right]^{1-\sigma}}{1-\sigma}
$$

donde el parámetro $\sigma$ mide el grado de concavidad de la función de utilidad. Cuando el parámetro $\sigma$ se aproxima a 1, obtenemos una función logarítmica:

$$
U\left(C_{t}, L_{t}\right)=\ln \left(C_{t}\right)+\gamma \ln \left(L_{t}\right)
$$


a la unidad:

$$
N_{t}=1 \quad \forall t
$$

Conocido (2), podemos definir (1):

$$
U\left(C_{t}, L_{t}\right)=N u\left(c_{t}, n_{l t}\right)=u\left(c_{t}, n_{l t}\right)
$$

donde $c_{t}$ es el nivel de consumo per capita.

La restricción temporal del individuo viene dada por:

$$
n_{s t}+n_{f t}+n_{l t}=1 \quad \forall t
$$

\subsubsection{Capital Humano}

Se asume que la educación formal incrementa la productividad del individuo tanto directamente (Lucas (1988,1993)), como indirectamente al incrementar el nivel de formación en la empresa (Altonji y Spletzer (1991)).

Se define $S_{t}$ como el nivel de educación formal del hogar y $s_{t}$ el correspondiente al individuo. Dado el supuesto (2), $S_{t}=S_{t}$. De forma análoga se define $T_{t}$ como el nivel de formación del hogar y $t_{t}$ es el nivel individual $\left(T_{t}=t_{t}\right)$.

Los hogares van a realizar una inversión en la escuela $\left(x_{t}\right)$, la cual va a depender del tiempo que destinan a estudiar en la escuela $\left(n_{s t}\right)$ y del nivel de educación en el momento de realizar la inversión $\left(s_{t}\right)$ :

$$
x_{t}=h\left(n_{s t}, s_{t}\right)=n_{s t} s_{t}
$$

para $h_{j}>0, \forall j=1,2$.

Tal y como se ha establecido anteriormente, el nivel de educación se deprecia a una tasa constante, lo que implica:

$$
\dot{s}_{t}=x_{t}-\delta s_{t}
$$

Combinando (5) y (6) se obtiene: 


$$
\dot{s}_{t}=\left[n_{s t}-\delta\right] s_{t}
$$

Por otro lado se asume que la productividad de los trabajadores es una función con rendimientos constantes a escala, que depende de $s_{t}$ y de $t_{t}$ :

$$
e\left(s_{t}, t_{t}\right)=s_{t}^{\phi} t_{t}^{1-\phi}
$$

donde $0<\phi<1, \frac{\partial e\left(s_{t}, t_{t}\right)}{\partial s_{t}}>0, \frac{\partial e\left(s_{t}, t_{t}\right)}{\partial t_{t}}>0, \frac{\partial^{2} e\left(s_{t}, t_{t}\right)}{\partial s_{t}^{2}} \leq 0$ y $\frac{\partial^{2} e\left(s_{t}, t_{t}\right)}{\partial t_{t}^{2}} \leq 0$.

\subsubsection{Restricción Financiera}

El individuo representativo tiene dos tipos de rentas: rentas del trabajo y rentas del capital.

Definiendo $w_{t}$ como el salario por unidad de tiempo, las rentas del trabajo vendrán dadas por:

$$
e\left(s_{t}, t_{t}\right) n_{f t} w_{t}
$$

Por otro lado el individuo puede ahorrar a través de activos financieros $\left(B_{t}=b_{t}\right)$. Se asume que no existen restricciones de liquidez, lo que implica que en Equilibrio: $B=K$, donde $K$ es el stock de capital físico. Estos activos de los que dispone el individuo se acumulan a través de la Inversión (I):

$$
\dot{B}_{t}=I_{t} \quad \forall t
$$

Los activos proporcionarán una rentabilidad al individuo igual a $b_{t} r_{t}$, donde $r_{t}$ es la rentabilidad del mercado de los activos, que coincide con el tipo de interés de mercado.

El nivel de riqueza del individuo se ve afectado por los beneficios de la empresa $\left(\Pi_{t}\right)$. Asumiendo que los individuos son propietarios de la empresa y que ésta se mueve en un mercado de competencia perfecta y ausencia de barreras de entrada, el nivel de beneficios será igual a cero. 
Considerando todos estos elementos, obtenemos la siguiente expresión para la restricción presupuestaria (Sala-i-Martin (1994):

$$
c_{t}+\dot{b}_{t}=w_{t} n_{f t}\left[s_{t}^{\phi} t_{t}^{1-\phi}\right]+r b_{t}
$$

\subsubsection{Problema de Maximización del Individuo Representativo}

El problema de maximización, en términos per capita, de un individuo representativo viene dado por:

$$
\begin{array}{ccc}
\operatorname{Max}_{\left\{c_{t}, n_{s t}, n_{f t}, s_{t}, b_{t}\right\}} & \int_{0}^{\infty} e^{-\rho t}\left[\ln \left(c_{t}\right)+\gamma \ln \left(n_{l t}\right)\right] d t & \\
\text { s.a. } & \dot{b}_{t}=w_{t}\left[s_{t}^{\phi} t_{t}^{1-\phi}\right] n_{f t}+r_{t} b_{t}-c_{t} & \\
n_{l t}=1-n_{s t}-n_{f t} & \\
\dot{s}_{t}=\left[n_{s t} s_{t}\right]-\delta s_{t} & \\
s(0)>0, b(0)>0 & \text { dados } \\
t_{t}, r_{t}, w_{t}>0 & \text { dados } \\
\lim _{t \rightarrow \infty}\left(b_{t} e^{\left[-\int_{0}^{t} r(v) d v\right]}\right) \geq 0 &
\end{array}
$$

Definiendo el vector de variables estado como: $Z=\left(s_{t}, b_{t}\right)$ y el vector de variables control como $\Theta=\left(c_{t}, n_{s t}, n_{f t}\right)$ se resuelve el problema (12) llegando a las ecuaciones (ver Anexo 1):

$$
\begin{gathered}
\frac{1-n_{s t}-n_{f t}}{\mu c_{t}}=\frac{1}{w_{t}\left[s_{t}^{\phi} t_{t}^{1-\phi}\right]} \\
\frac{\dot{c}_{t}}{c_{t}}=r_{t}-\rho \\
\frac{\lambda_{1 t}}{\lambda_{2 t}}=\frac{s_{t}}{w_{t}\left[s_{t}^{\phi} t_{t}^{1-\phi}\right]}
\end{gathered}
$$

donde (13) representa el ratio de utilidades marginales entre ocio y consumo, (14) la senda de crecimiento del capital per capita en 
equilibrio (la cual va a ser constante) y (15) el ratio de los multiplicadores dinámicos. ${ }^{3}$

\subsection{Empresa}

\subsubsection{Formación en la Empresa}

En primer lugar la empresa debe decidir el nivel de formación del que proveer al individuo para conseguir el uso más eficiente de la tecnología disponible. Se asume que esta formación depende del nivel educativo del individuo, ya que este último influye tanto en la velocidad de adquisición de la formación (como señalan Einarsson y Marquis (1999)) como en la cantidad de formación (tal y como han señalado entre otros Altonji y Spletzer (1991)).

Conocido $n_{f t}$, las empresas distribuyen este tiempo de la forma siguiente:

$$
n_{f t}=n_{t t}+n_{p t} \quad \forall t
$$

Se asume que la empresa soporta todos los costes de formación, de manera que paga un salario por el tiempo total que destina el trabajador a la empresa $\left(n_{f t}\right)$, a pesar de que el tiempo productivo es sólo una parte de ese tiempo total $\left(n_{p t}\right) .4$

Se define $z_{t}$ como la inversión que realiza la empresa en formación, la cual va a ser función del nivel de educación $\left(s_{t}\right)$, del tiempo dedicado a formación $\left(n_{t t}\right)$ y del nivel de esta última en el momento $\left(t_{t}\right)$ :

$$
z_{t}=g\left(n_{t t}, t_{t}, s_{t}\right)
$$

donde se asume que $\frac{\partial g\left(n_{t t}, t_{t}, s_{t}\right)}{\partial n_{t t}}>0, \frac{\partial g\left(n_{t t}, t_{t}, s_{t}\right)}{\partial t_{t}}>0$ y $\frac{\partial g\left(n_{t t}, t_{t}, s_{t}\right)}{\partial s_{t}}>0$.

\footnotetext{
$3 \lambda_{1 t}$ es el multiplicador asociado a los activos y $\lambda_{2 t}$ el asociado a la educación.

${ }^{4}$ No obstante, tal y como han puesto de manifiesto algunos autores, decir que es la empresa la que sustenta el coste de la formación es inadecuado, ya que con el tiempo son los trabajadores los que devuelven el préstamo recibiendo un salario inferior a su productividad marginal.
} 
La ecuación dinámica que determina la acumulación de formación vendrá dada por la expresión:

$$
\dot{t}_{t}=z_{t}-\delta t_{t}
$$

Combinando (17)y (18) se obtiene:

$$
\dot{t}_{t}=g\left(n_{t t}, t_{t}, s_{t}\right)-\delta t_{t}
$$

Por último se define $H^{S}\left(=s_{t} n_{p t}\right)$ como las unidades efectivas de educación en la producción, y $H^{T}\left(=t_{t} n_{p t}\right)$ como las unidades efectivas de formación en la producción.

\subsubsection{Problema de la Empresa}

La empresa maximiza una función de beneficios intertemporal, actualizando al tipo de interés de mercado $r_{t}$.

El stock de capital físico se deprecia a una tasa constante $\delta_{K}$. De acuerdo con el trabajo de Sala-i-Martin (1994), los propietarios del capital son los hogares, los cuales alquilan cada una unidad de capital a las empresas a un precio $R_{t}$. Dado que el rendimiento de los activos $\left(B_{t}\right)$ debe ser igual al rendimiento del capital $\left(K_{t}\right)$, es claro que: $r_{t}=R_{t}-\delta_{K}$.

La función de beneficios de la empresa representativa vendrá dada por la expresión:

$$
\Pi_{t}=F\left(A_{t}, K_{t}, H_{t}^{S}, H_{t}^{T}\right)-\left(r_{t}+\delta_{K}\right) K_{t}-w_{t} n_{f t}
$$

donde $A_{t}$ es el stock de tecnología.

Por otro lado se asume que la función de producción queda representada como:

$$
Y_{t}=F\left(A_{t}, K_{t}, H_{t}^{S}, H_{t}^{T}\right)=A_{t} F\left(K_{t}, H_{t}^{S}, H_{t}^{T}\right)
$$

siendo una función homogénea de grado uno, con productividad marginal de los factores positiva pero decreciente y que satisface las 
condiciones de Inada. ${ }^{5}$

Se asume una forma funcional de la función de producción definida en (21), en concreto una función CES dentro de una CES: 6

$$
F\left(A_{t}, K_{t}, H_{t}^{S}, H_{t}^{T}\right)=A_{t}\left\{\mu\left[K_{t}^{\varphi}+\left(H_{t}^{T}\right)^{\varphi}\right]^{\sigma / \varphi}+\left(H_{t}^{S}\right)^{\sigma}\right\}^{1 / \sigma}
$$

donde $\sigma$ va a determinar la elasticidad de sustitución entre el capital físico y la educación, así como entre esta última y la formación en el puesto de trabajo. Por su parte $\varphi$ va a determinar la elasticidad de sustitución entre el stock de capital físico y el nivel de formación dentro de la empresa.

Para simplificar se asume que la tecnología no cambia, es decir $A_{t}=A \quad \forall t$.

El problema de maximización de la empresa representativa

5 Las condiciones de Inada tal como Sala-i-Martin (1994) muestra en la página 47 requieren para el capital:

$$
\begin{aligned}
& \lim _{K \rightarrow \infty} \frac{\partial F\left(A, K, H^{S}, H^{T}\right)}{\partial K}=0 \\
& \lim _{K \rightarrow 0} \frac{\partial F\left(A, K, H^{S}, H^{T}\right)}{\partial K}=\infty
\end{aligned}
$$

y así para cada uno de los factores de producción $H^{S}$ y $H^{T}$.

${ }^{6}$ Utilizamos una función CES (Constant Elasticity of Substitution) del tipo:

$$
F\left(B_{t}, K_{t}, H_{t}^{S}, H_{t}^{T}\right)=B_{t}\left\{b\left[K_{t}^{\varphi}+\left(H_{t}^{T}\right)^{\varphi}\right]^{\sigma / \varphi}+(1-b)\left(H_{t}^{S}\right)^{\sigma}\right\}^{1 / \sigma}
$$

De acuerdo con la utilizada en los modelos de crecimiento económico (Sala-iMartin (1994, página 35)). Donde los parámetros cumplen: $0<b<1,-\infty<\sigma<1,0<\varphi<1$.

Para evitar problemas de multicolinealidad transformamos la función de manera que definiremos: $\mu=b /(1-b)$, donde $0<\mu<\infty$. Así, llegamos a la función:

donde:

$$
F\left(A_{t}, K_{t}, H_{t}^{S}, H_{t}^{T}\right)=A_{t}\left\{\mu\left[K_{t}^{\varphi}+\left(H_{t}^{T}\right)^{\varphi}\right]^{\sigma / \varphi}+\left(H_{t}^{S}\right)^{\sigma}\right\}^{1 / \sigma}
$$

$$
A_{t}=B(1-b)^{1 / \sigma}
$$


viene dado por:

$$
\begin{array}{cc}
\underset{\left\{K_{t}, n_{p t}, n_{t}, t_{t}\right\}}{\operatorname{sax}} & \int_{0}^{\infty} e^{-r t}\left[F\left(A, K_{t}, H_{t}^{S}, H_{t}^{T}\right)-\left(r_{t}+\delta_{K}\right) K_{t}-w_{t} n_{f t}\right] d t \\
\text { s.a. } \quad F\left(A, K_{t}, H_{t}^{S}, H_{t}^{T}\right)=A\left\{\mu\left[K_{t}^{\varphi}+\left(H_{t}^{T}\right)^{\varphi}\right]^{\sigma / \varphi}+\left(H_{t}^{S}\right)^{\sigma}\right\}^{1 / \sigma} \\
n_{f t}=n_{t t}+n_{p t} \\
\dot{t}_{t}=g\left(n_{t t}, t_{t}, s_{t}\right)-\delta t_{t} \\
t(0)>0 \quad \text { dado } \\
s_{t}, n_{f t}, w_{t}>0 \quad \text { dados }
\end{array}
$$

donde la variable estado es $t_{t}$ y el vector de variables control viene definido por $\Xi=\left(K_{t}, n_{p t}, n_{t t}\right)$. Para completar el problema se impone la condición de vaciado de mercado:

$$
C_{t}+I_{t}=Y_{t}=F\left(A, K_{t}, H_{t}^{S}, H_{t}^{T}\right)
$$

Resolviendo (23) se obtiene (ver Anexo 2):

$$
\begin{gathered}
\frac{\partial F\left(A, K_{t}, H_{t}^{S}, H_{t}^{T}\right)}{\partial K_{t}}=\left(r_{t}+\delta_{K}\right) \\
\frac{\partial F\left(A, K_{t}, H_{t}^{S}, H_{t}^{T}\right)}{\partial H_{t}^{T}} t_{t}+\frac{\partial F\left(A, K_{t}, H_{t}^{S}, H_{t}^{T}\right)}{\partial H_{t}^{S}} S_{t}=w_{t}
\end{gathered}
$$

La ecuación (25) representa la condición clásica bajo la cual la productividad marginal del capital es igual a la suma del coste del factor capital $(r)$ y la depreciación del mismo $\left(\delta_{K}\right)$.

A partir de (26) pueden obtenerse las siguientes conclusiones. Por un lado, para un nivel de formación dado, cuanto mayor sea el nivel de educación de un trabajador mayor será el salario por hora de equilibrio. Por otro lado, dado el nivel de educación, cuanto mayor es la formación que reciben los trabajadores en su puesto de trabajo, mayor es el salario por hora que perciben en equilibrio. 
A modo de ejemplo en el Anexo 9 se estima la función de producción (22) para contrastar si se cumple que la complementariedad del capital físico $(K)$ es mayor con las unidades efectivas de formación $\left(H^{T}\right)$ que con las unidades efectivas de educación en la escuela $\left(H^{S}\right)$. De igual modo, estimando la función de producción podremos ver el peso que tiene cada una de las fuentes de capital humano en el nivel de producción de la economía.

\section{Complementariedad entre las Distintas Fuentes de Crecimiento: Capital Físico y Capital Humano}

A la hora analizar las fuentes de crecimiento económico de una economía, es importante conocer el grado de complementariedad entre capital físico y capital humano. Dicha complementariedad puede variar entre los distintos sectores de actividad de una economía, de manera que las políticas económicas dirigidas a incentivar el crecimiento del país, deberán ser específicas de cada sector.

Serrano-Martínez (2003) analiza para la economía española como el capital humano varía entre los distintos sectores de actividad, si bien no distingue entre sus distintas fuentes, ni entra a valorar el grado de complementariedad de este con el capital físico.

Siguiendo la línea de este trabajo, en esta sección se estudia la relación entre las distintas formas de capital utilizando datos de sectores de actividad de la economía española. Para la consecución de este objetivo se utilizan datos del BBVA y Bancaja, para el año 1993. ${ }^{7}$

Antes de comenzar el análisis de los datos, es conveniente introducir una serie de conceptos.

En primer lugar, definimos la elasticidad de sustitución entre las dos formas de acumulación de capital humano como $\varepsilon_{H^{s}, H^{T}}$, que coincide con la elasticidad de sustitución entre la educación y el capital físico, $\varepsilon_{H^{s}, K}$ (ver Anexo 4):

$$
\varepsilon_{H^{S}, H^{T}}=\varepsilon_{H^{S}, K}=\left[\frac{1}{1-\sigma}\right]
$$

y la elasticidad de sustitución entre el capital físico y formación en la empresa (ver Anexo 5) como:

\footnotetext{
${ }^{7}$ Ver Anexo 3 para la definición de variables.
} 


$$
\varepsilon_{H^{T}, K}=\left[\frac{1}{1-\varphi}\right]
$$

En segundo lugar, se puede definir el skill premium $(\Gamma)$ como el cociente entre la productividad marginal de la educación formal y la productividad marginal de la formación en el puesto de trabajo. Utilizando (22) es fácil de comprobar que queda expresado (ver Anexo 6) como:

$$
\Gamma=\frac{1}{\mu}\left[K_{t}^{\varphi}+\left(H_{t}^{T}\right)^{\varphi}\right]^{1-\left(\frac{\sigma}{\varphi}\right)}\left[\frac{\left(H_{t}^{T}\right)^{1-\varphi}}{\left(H_{t}^{S}\right)^{1-\sigma}}\right]
$$

Tomando (29), se puede ver el efecto que tiene un incremento del capital físico en $\Gamma$ :

$$
\frac{\partial \Gamma}{\partial K}=\frac{1}{\mu}(\varphi-\sigma)\left[K_{t}^{\varphi}+\left(H_{t}^{T}\right)^{\varphi}\right]^{-\left(\frac{\sigma}{\varphi}\right)}\left[\frac{\left(H_{t}^{T}\right)^{1-\varphi}}{\left(H_{t}^{S}\right)^{1-\sigma}}\right]
$$

Proposición 1: Un incremento del stock de capital físico provoca un aumento (disminución) de la productividad marginal de la educación en la escuela respecto a la productividad marginal de la formación siempre que $\varphi>\sigma(\varphi<\sigma)$, para valores de $\varphi, \sigma>0$.

Pasamos ahora a los datos. Siguiendo otros estudios previos (como el de Serrano-Martínez (2003)) se consideran cinco sectores de actividad (Cuadro 1).

Cuadro 1. Ratios de Factores de Producción respecto del VAB (coste de los factores). Base: 1986.

\begin{tabular}{lcccc}
\hline Sectores & $\mathrm{K} / \mathrm{Y}$ & $\mathrm{Hs} / \mathrm{Y}$ & $\mathrm{Ht} / \mathrm{Y}$ & $\mathrm{Ht} / \mathrm{Hs}$ \\
\hline Agricultura & 1,56 & $9,91 \mathrm{E}-08$ & $3,57 \mathrm{E}-09$ & 0,038 \\
Construcción & 0,29 & $1,58 \mathrm{E}-07$ & $8,92 \mathrm{E}-09$ & 0,057 \\
Energía & 0,89 & $1,30 \mathrm{E}-07$ & $6,39 \mathrm{E}-09$ & 0,979 \\
Industria & 1,31 & $1,46 \mathrm{E}-07$ & $1,90 \mathrm{E}-08$ & 0,130 \\
Servicios & 3,66 & $1,67 \mathrm{E}-07$ & $2,05 \mathrm{E}-08$ & 0,123 \\
\hline
\end{tabular}

Fuente: Elaboración propia a partir de los datos del BBVA y Bancaja (año 1993) 
El Cuadro 1 muestra que el sector con mayor capitalización es el sector servicios, seguido de la agricultura y de la industria. Por contra el menos capitalizado es la construcción. ${ }^{8}$

Como puede observarse, el sector servicios presenta los mayores niveles tanto de educación en la escuela como formación en el lugar de trabajo. Por el contrario, encontramos los niveles más bajos de capital humano en el sector agrícola. Resulta importante señalar que el peso de la formación en el puesto de trabajo con respecto a la adquirida en la escuela, es significativamente mayor en el sector de la energía.

Analizando las correlaciones entre los distintos factores de producción para cada uno de los sectores de actividad, puede observarse que en la mayoría de los casos la correlación entre capital físico y educación formal es mayor que la correspondiente a capital físico y formación en la empresa, lo que sugiere un mayor grado de complementariedad entre capital físico y educación (ver Anexo 8).

No obstante para corroborar si esto último es cierto, resulta necesario estimar los parámetros del modelo, y más concretamente la elasticidad de sustitución entre el capital físico y las distintas formas de capital humano. Hemos llevado a cabo a modo de ejemplo una estimación de la función de producción (ver Anexo 9).

\section{Capital Humano y Salarios: Un Estudio Basado en el Panel de Hogares}

El objeto de esta sección es ver el peso de la educación en la escuela y la formación en el puesto de trabajo en la determinación de los salarios.

Para ello se utilizan datos españoles extraídos del Panel de Hogares Europeo (PHOGUE, 1995-2001). Se selecciona una muestra de individuos con edades comprendidas entre 16 y 64 años, que trabajan como asalariados. Estimamos un modelo de regresión donde la variable dependiente es el logaritmo del salario bruto por hora y donde se incluyen como variables explicativas tanto características individuales como características del puesto de trabajo. Los resultados aparecen reflejados en el Cuadro 2.

\footnotetext{
8 Los datos están recogidos en el Anexo 7.
} 


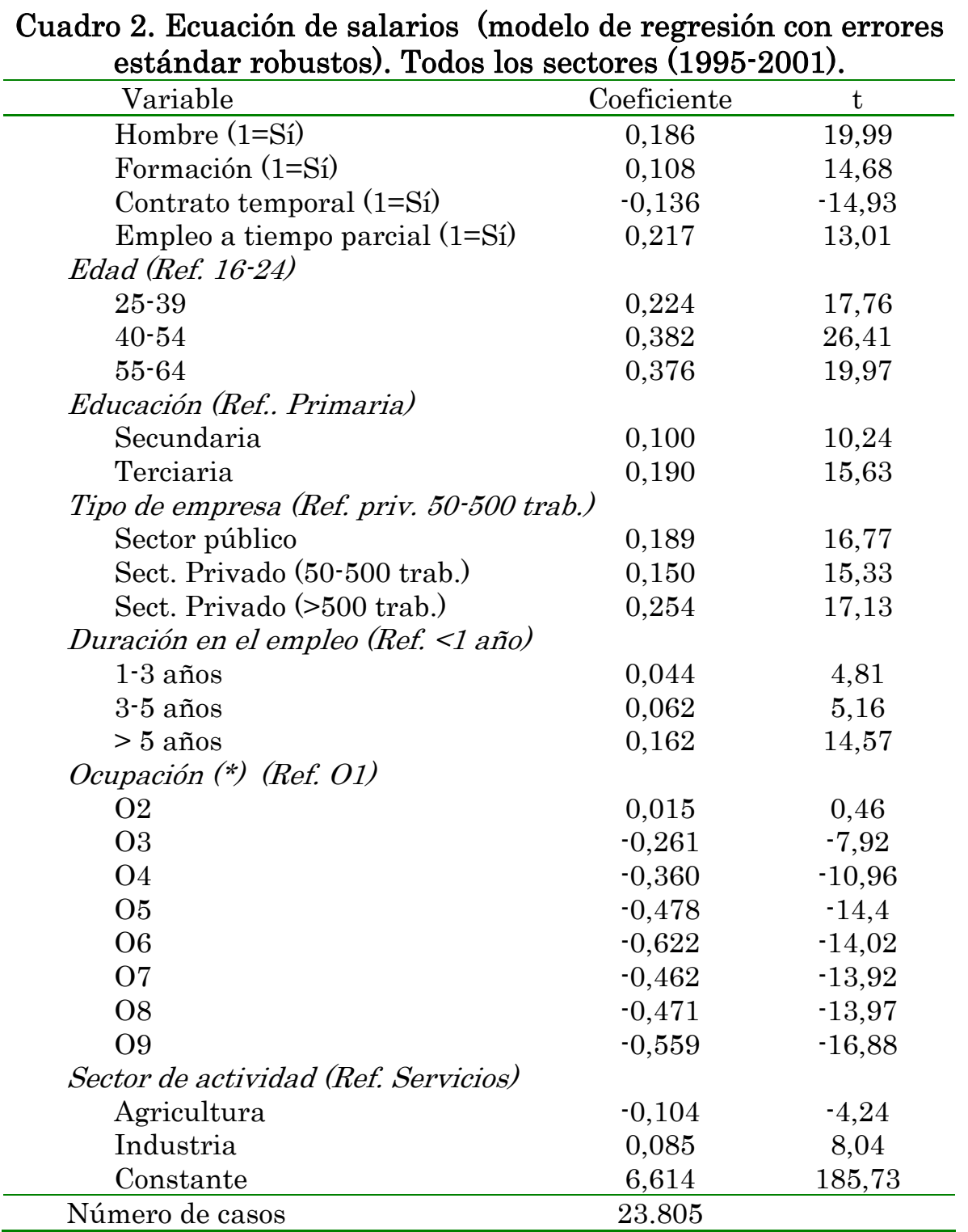

(*) O1: Dirección de las empresas y de las administraciones públicas; O2: Técnicos y profesionales científicos e intelectuales; O3: Técnicos y profesionales de apoyo; O4: Empleados de tipo administrativo; O5: Trabajadores de los servicios de restauración, personales, protección y vendedores de comercios; 06: Trabajadores cualificados en la agricultura y en la pesca; 07: Artesanos y trabajadores cualificados de las industrias manufactureras, la construcción, y la minería, excepto los operadores; 08: Operadores de instalaciones y maquinaria, y montadores; O9: Trabajadores no cualificados.

Fuente: Panel de Hogares de la Unión Europea (1995-2001). 
Como se desprende de los resultados del Cuadro 2, las dos formas de capital humano (educación en la escuela y formación) afectan de manera positiva y significativa las ganancias salariales de los trabajadores.

En lo concerniente a la educación formal y tomando como referencia el nivel de estudios primario, se observa que el tener educación terciaria aumenta las ganancias salariales en un 19\%. Por su parte los individuos que reciben formación por parte de la empresa, ven incrementar su salario bruto un $10 \%$ con respecto a aquellos individuos que no reciben esta formación. Estos resultados están en línea con los obtenidos en trabajos previos como los de Psacharopoulos y Patrinos (2002), Brunello y Comi (2000), Harmon et al. (2001) y De la Fuente y Jimeno (2005).

Por otro lado, las estimaciones están en sintonía con lo analizado en la Sección 3, donde veíamos como el salario de equilibrio de individuo representativo venía determinado por su nivel de educación y su formación en el puesto de trabajo (ver ecuación (26)).

Respecto al efecto del resto de variables incluidas en el análisis, los resultados son similares a los obtenidos por trabajos previos en la literatura. Por un lado las mujeres y los jóvenes tienen unas ganancias salariales significativamente menores. Respecto al tipo de contrato y tamaño de la empresa, destacar que los trabajadores temporales tienen menores ganancias salariales. Así mismo los trabajadores en empresas grandes son los que obtienen ganancias salariales mayores. La duración del empleo también es un resultado predecible, aquellos individuos que más tiempo han permanecido en su puesto de trabajo perciben salarios mayores. Por último, en lo que concierne al sector de actividad, los trabajadores del sector industrial perciben mayores salarios que los del sector servicios, mientras que en la agricultura los salarios son menores que en este último.

\section{Conclusiones}

El objetivo de este trabajo se ha centrado, por un lado, en analizar el papel de las distintas fuentes de capital humano (educación en la escuela y formación en el puesto de trabajo) como motores del crecimiento económico y, por otro, en observar el efecto de estos factores en la determinación de los salarios de los trabajadores.

En lo que se refiere al primer aspecto, los resultados obtenidos a partir de los datos de la economía española revelan que existen diferencias sectoriales significativas. Así, mientras que en el sector agrícola y en el sector servicios la educación en la escuela y la formación 
en el trabajo aparecen como factores con un alto grado de sustitución, en el resto de sectores (construcción, energía e industria) estas formas de capital humano tienen un carácter complementario. Una situación similar se observa en la relación entre el capital físico y educación en la escuela.

Como otros resultados interesantes podemos señalar los siguientes: por un lado, en los sectores construcción, energía e industria el grado de complementariedad entre capital físico y formación en el trabajo es mayor que el correspondiente al capital físico y a la educación en la escuela. Y por otro lado, en el sector servicios se encuentra que todos los factores productivos son sustitutivos.

Con respecto al segundo punto, el efecto de la educación y la formación en el trabajo, sobre las estimaciones de la ecuación de salarios a partir de los datos del Panel de Hogares (1995-2001) para la economía española, corroboran el efecto positivo de ambas formas de acumulación de capital humano sobre la determinación de salarios.

\section{Bibliografía}

Acemoglu, D. and J.-S., Pischke (1999b), "Beyond Becker: Training in imperfect labour markets", Economic Journal 109 (February).

Alan, E. (1993), "Job-Related education and training: their impact on Earnings", Monthly Labor Review 116, pp. 21-38

Altonji, J. G. and Spletzer, J. R. (1991), "Worker characteristics, job characteristics, and the receipt of on-the-job training", Industrial and Labor Relations Review 45, pp. 58-79.

Barro, R.J. and J.-W. Lee (1993), "International comparisons of educational attainment", Journal of Monetary Economics 32, pp. 363394

Barron, J. M., Black, D. A. y Loewenstein, M. A. (1993), "Gender differences in training, capital and wages", Journal of Human Resources, 28(2), pp. 343-365.

Becker, G. (1962), "Investment in human capital: A theoretical analysis", Journal of Political Economy, 70, pp. 9-49.

Benhabib J. and M.M. Spiegel (1994), "The role of human capital in economic development. Evidence from aggregate cross-country data", Journal of Monetary Economics 34, pp. 143-173.

Brunello, G. (2001),”On the complementarity between education and 
training in Europe", IZA Discussion Paper, Nº309 (June, 2001).

Bruenllo, G. and Comi, S. (2000), "Education and earnings growth: Evidence from 11 European countries", IZA Discussion Paper, N ${ }^{\circ} 140$ (April, 2000).

Card, D. (1995), "Earnings, schooling and ability revisited", Research in Labour Economics, 14, pp. 23-48.

Cass, D. (1965), "Optimum growth in an aggregate model of capital accumulation", Review of Economic Studies, 32, pp. 233-240.

Cohen, D. y Soto, M.(2001), "Growth and human capital : good data, good results". CEPR Discussion Paper, $\mathrm{N}^{\mathrm{o}} 3025$

De la Fuente, A. y Da Rocha, J.M. (1996), "Capital Humano y Crecimiento Económico. Evidencia Empírica y situación española en relación a la OCDE. Moneda y Crédito 203, pp. 43-84.

De la Fuente, A., y Jimeno, J.F. (2005), "The private and fiscal returns to schooling and the effect of public policies on private incentives to invest in education. A general framework and results for the EU", CESIfo mimeo

De la Fuente, A., y Doménech, F. (2006), "Human capital in growth regressions: how much difference does data quality make?", Journal of the European Economic Association, 4(1), pp. 1-36.

Denison, E. (1962), The Sources of Growth in the US, Committee for Economic Development, New York.

Duncan, G. and Hoffman, S.D. (1981), "The incidence and wage effects of overeducation", Economics of Education Review, 1(1), pp. 75-86.

Einarsson, T. y Marquis, M.H. (1999), "Formal Training, On-the-job Training and the Allocation of Time", Journal of Macroeconomics, 21(3), pp. $423-442$.

Hall, R.E. y C.I. Jones (1999), "Why do some countries produce so much more output per worker than others?", Quaterly Journal of Economics 114 (1), pp. 83-116.

Harmon, C., Walker, I. y Westergaard-Nielsen, N. (2001), "Introduction", en Harmon, C., Walker, I. y Westergaard-Nielsen, N., eds, Education and Earnings in Europe. A cross country analysis of the returns to education, Edward Elgar, Cheltenham, pp. 1-37.

Helpman, E. y A.Rangel (1998), "Adjusting to a new technology: Experience and training", Mimeo, Department of Economics, Stanford 
University.

Kass, L. y Stefan, Z. (2007), "Human capital and growth cycles", Economic Theory, 31, pp. 19-33.

Koopmans, T.C. (1965), "On the concept of optimal economic growth", in The Econometric Approach to Development Planning, Amsterdam: North Holland

Lucas, R.E., Jr. (1988), "On the mechanics of economic development", Journal of Monetary Economics, 22, pp. 3-42.

Lucas, R.E., Jr. (1993), "Making a miracle", Econometrica, 61(2), pp. 251-272.

Lynch, L.M. (1993), "The Economics of youth training in the United States", Economic Journal, 103 (420), pp. 1292-1302.

Mankiw, N.G., D.Romer y D.N. Weil (1992), "A contribution to the empirics of economic growth", Quarterly Journal of Economics, 107, pp. 407-437.

McGrattan, E.R. y J.A. Schmitz, Jr., (1998), "Explaining Cross-Country Income Differences", Federal Reserve Bank of Minneapolis, Research Department, Staff Report 250.

Mincer, J. (1974), Schooling, experience and earnings. New York: NBER.

O'neill, D. (1995), "Education and income growth: Implications for crosscountry inequality", Journal of Political Economy 103(6), pp. 1289-1301.

Parente, S.L. y E.C. Prescott (1993), "Changes in the wealth of nations", Federal Reserve Bank of Minneapolis Quarterly Review 17, pp. 3-16.

Prescott, E.C. (1997), "Needed: A theory of total factor productivity", Federal Reserve Bank of Minneapolis, Research Department, Staff Report 242.

Psacharopoulos, G. (1981), "Returns to education: an updated international comparison", Comparative Education, 17, pp.321-341.

Psacharopoulos, G. (1985), "Returns to education: A further international update and implications", Journal of Human Resources, 20(4), pp. 1325-1343.

Psacharopoulos, G., y Patrinos, H. (2002), "Returns to investment in education: A further update", World Bank Policy Research, Working Paper 2881, (September, 2002). 
Rebelo, S. (1991), "The long-run analysis and lon-run growth", Journal of Political Economy, 99, pp. 500-521.

Romer, P.M (1986), "Increasing returns and long-run growth", Journal of Political Economy, 94(5), pp. 1002-1037.

Romer, P.M. (1990), "Endogenous technological change", Journal of Political Economy, 98(2), pp. 71-102.

Sala-i-Martin, X. (1994), Apuntes de Crecimiento Económico. Antoni Bosch Editor, Barcelona.

Schultz, W (1960), "Capital formation by education", Journal of Political Economy, 68(6), pp. 571-583.

Schultz, W (1961), "Investment in human capital", American Economic Review, 51(1), pp. 1-17.

Serrano-Martínez, L. (2003), "Human Capital Externalities: A sectorialRegional Application for Spain", Economic Working Paper at Centro de Estudios Andaluces No. E2003/06.

Tamura, R. (1991)," Income convergence in an endogenous growth model", Journal of Political Economy 99(3), pp. 522-540.

Uzawa, H. (1965): "Optimum Technical Change in an Aggregative Model of Economic Growth." International Economic Review, 6, pp. 1831.

Temple, J. (2001), "Growth effects of education and social capital in the OECD countries", OECD Economic Studies, 33 (2), pp. 57-101

Willis, Robert J. (1986), "Wage determinants: A survey and reinterpretation of human capital earnings function", en Orley Ashenfelter y Richard Layard, eds., Handbook of Labour Economics, Vol I, pp 525-601. 


\section{Anexos}

\section{Anexo 1}

Se resuelve el problema (12) de los hogares planteando el hamiltoniano:

$$
\begin{gathered}
H\left(c_{t}, n_{s t}, n_{f t}, b_{t}, s_{t} ; \lambda_{1 t}, \lambda_{2 t}\right)= \\
e^{-\rho t}\left[\ln \left(c_{t}\right)+\gamma \ln \left(1-n_{s t}-n_{f t}\right)\right]+ \\
+\lambda_{1 t}\left[w_{t} s_{t}^{\phi} t_{t}^{1-\phi} n_{f t}+r_{t} b_{t}-c_{t}\right]+\lambda_{2 t}\left[\left[n_{s t} s_{t}\right]-\delta s_{t}\right]
\end{gathered}
$$

donde $\lambda_{1 t}$ y $\lambda_{2 t}$ son los multiplicadores dinámicos asociados a cada una de las restricciones dinámicas.

Calculando las condiciones de primer orden se obtienen las siguientes expresiones:

$$
\begin{gathered}
\frac{\partial H\left(c_{t}, n_{s t}, n_{f t}, b_{t}, s_{t} ; \lambda_{1 t}, \lambda_{2 t}\right)}{\partial c_{t}}=0 \Rightarrow e^{-\rho t}\left[\frac{1}{c_{t}}\right]-\lambda_{1 t}=0 \\
\frac{\partial H\left(c_{t}, n_{s t}, n_{f t}, b_{t}, s_{t} ; \lambda_{1 t}, \lambda_{2 t}\right)}{\partial n_{s t}}=0 \Rightarrow-e^{-\rho t}\left[\frac{\gamma}{1-n_{s t}-n_{f t}}\right]+\lambda_{2 t}\left[s_{t}\right]=0 \\
\frac{\partial H\left(c_{t}, n_{s t}, n_{f t}, b_{t}, s_{t} ; \lambda_{1 t}, \lambda_{2 t}\right)}{\partial n_{f t}}=0 \Rightarrow \\
\frac{\partial H\left(c_{t}, n_{s t}, n_{f t}, b_{t}, s_{t} ; \lambda_{1 t}, \lambda_{2 t}\right)}{\partial b_{t}\left[\frac{\mu}{1-n_{s t}-n_{f t}}\right]+\lambda_{1 t}\left[w_{t}\left[s_{t}^{\phi} t_{t}^{1-\phi}\right]\right]=0} \\
\frac{\partial H\left(c_{t}, n_{s t}, n_{f t}, b_{t}, s_{t} ; \lambda_{1 t}, \lambda_{2 t}\right)}{\partial s_{t}}=-\lambda_{1 t}\left[r_{t}\right]=-\dot{\lambda}_{1 t} \\
\lambda_{1 t}\left[\begin{array}{c}
\left.w_{t} n_{f t}\left[\phi \frac{t_{t}^{1-\phi}}{s_{t}^{1-\phi}}\right]\right]+\lambda_{2 t}\left[n_{s t}-\delta\right]=-\dot{\lambda}_{2 t} \\
\lim _{t \rightarrow \infty} b_{t} \lambda_{1 t}=0 \\
\lim _{t \rightarrow \infty} s_{t} \lambda_{2 t}=0
\end{array}\right.
\end{gathered}
$$


Combinando la condición (32) y (33) se obtiene (13). Así mismo de la ecuación (32) junto con la (35) se obtiene (14).

Por otro lado tomando (33) y (34) se deriva la expresión (15).

\section{Anexo 2}

Tomando el problema en (23), se plantea el hamiltoniano:

$$
\begin{aligned}
H\left(K_{t}, n_{p t}, n_{t t}, t_{t} ; p_{1 t}\right) & =e^{-r t}\left[A\left\{\mu\left[K_{t}^{\varphi}+\left(H_{t}^{T}\right)^{\varphi}\right]^{\sigma / \varphi}+\left(H_{t}^{S}\right)^{\sigma}\right\}^{1 / \sigma}\right]- \\
-e^{-r t} & {\left[\left(r_{t}+\delta_{K}\right) K_{t}+w_{t}\left(n_{t t}+n_{p t}\right)\right]+} \\
& +p_{1 t}\left[g\left(n_{t t}, t_{t}, s_{t}\right)-\delta t_{t}\right]
\end{aligned}
$$

donde $p_{l t}$ es el multiplicador dinámico asociado a la ecuación de acumulación de formación.

Se calculan las condiciones de primer orden:

$$
\begin{aligned}
& \frac{\partial H\left(K_{t}, n_{p t}, n_{t t}, t_{t} ; p_{1 t}\right)}{\partial K_{t}}=0 \Rightarrow e^{-r t}\left[\frac{\partial F\left(A, K_{t}, H_{t}^{S}, H_{t}^{T}\right)}{\partial K_{t}}-\left(r_{t}+\delta_{K}\right)\right]=0 \\
& \frac{\partial H\left(K_{t}, n_{p t}, n_{t t}, t_{t} ; p_{1 t}\right)}{\partial n_{p t}}=0 \Rightarrow \\
& e^{-r t}\left[\frac{\partial F\left(A, K_{t}, H_{t}^{S}, H_{t}^{T}\right)}{\partial H_{t}^{T}} t_{t}+\frac{\partial F\left(A, K_{t}, H_{t}^{S}, H_{t}^{T}\right)}{\partial H_{t}^{S}} s_{t}-w_{t}\right]=0 \\
& \frac{\partial H\left(K_{t}, n_{p t}, n_{t t}, t_{t} ; p_{1 t}\right)}{\partial n_{t t}}=0 \Rightarrow-e^{-r t}\left[w_{t}\right]+p_{1 t}\left[\frac{\partial g\left(n_{t t}, t_{t}, s_{t}\right)}{\partial n_{t t}}\right]=0 \\
& \frac{\partial H\left(K_{t}, n_{p t}, n_{t t}, t_{t} ; p_{1 t}\right)}{\partial t_{t}}=-\dot{p}_{1 t} \Rightarrow \\
& e^{-r t}\left[\frac{\partial F\left(A, K_{t}, H_{t}^{S}, H_{t}^{T}\right)}{\partial H_{t}^{T}} n_{p t}\right]+p_{1 t}\left[\frac{\partial g\left(n_{t t}, t_{t}, s_{t}\right)}{\partial t_{t}}-\delta\right]=-\dot{p}_{1 t} \\
& \lim _{t \rightarrow \infty} t_{t} p_{1 t}=0
\end{aligned}
$$




\section{Anexo 3}

Definición de las variables objeto de estudio:

- Variable de producción (VAB), producción de una determinada comunidad autónoma en 1993 a precios constantes de 1986.

- Stock de capital neto privado $(K)$ a precios constantes 1986.

- Educación formal en la escuela $\left(H^{S}\right)$, que se define como $H_{t} S^{S}=L_{t} s t n_{p t}$, es decir, el producto del numero de trabajadores $\left(L_{t}\right)$, por el tiempo que destinan a educarse en la escuela $\left(s_{t}\right)$ y por las horas que posteriormente van a trabajar en la empresa $\left(n_{p t}\right)$. Para esta variable tomamos el número de individuos con al menos educación secundaria, calculamos su porcentaje por sector y comunidad y luego se aplica el porcentaje al total de asalariados. Por lo que estamos tomando como referencia siempre el total de individuos que trabajan por sector $\mathrm{y}$ comunidad.

- Formación en el trabajo $\left(H^{T}\right)$. De igual modo, calculamos número de individuos que han recibido formación en el trabajo, se calcula el porcentaje por sector y comunidad y luego se aplica el porcentaje al total de asalariados.

\section{Anexo 4}

Se define la elasticidad de sustitución entre educación y formación como:

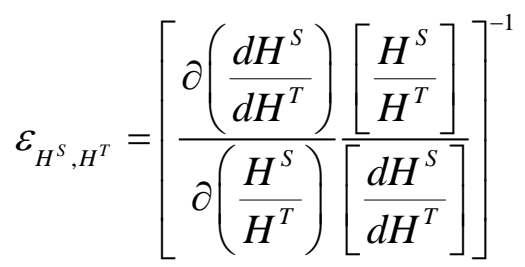

y tomando nuevamente (22) obtenemos (27). De forma análoga la elasticidad de sustitución entre el capital físico y la educación formal en la escuela será:

$$
\left.\varepsilon_{H^{S}, K}=\left[\frac{\partial\left(\frac{d H^{S}}{d K}\right)}{\partial\left(\frac{H^{S}}{K}\right)} \frac{H^{S}}{K}\right]\right]^{-1}
$$

donde $\frac{d H^{S}}{d K}$ no es más que la pendiente de la curva isocuanta, para un 
valor fijado de $H^{T}$. Tomando la ecuación (22) se obtiene (27).

\section{Anexo 5}

De forma análoga a como se ha definido la elasticidad de sustitución en (45) y (46) se demuestra (28).

\section{Anexo 6}

Sea:

$$
\Gamma=\frac{\frac{\partial F\left(A_{t}, K_{t}, H_{t}^{S}, H_{t}^{T}\right)}{\partial H_{t}^{S}}}{\frac{\partial F\left(A_{t}, K_{t}, H_{t}^{S}, H_{t}^{T}\right)}{\partial H_{t}^{T}}}
$$

Se calcula la productividad marginal de cada una de las formas de acumular capital humano. Usando (22) se obtiene:

$$
\begin{aligned}
& \frac{\partial F\left(A_{t}, K_{t}, H_{t}^{S}, H_{t}^{T}\right)}{\partial H_{t}^{S}}=A_{t}\left\{\mu\left[K_{t}^{\varphi}+\left(H_{t}^{T}\right)^{\varphi}\right]^{\sigma / \varphi}+\left(H_{t}^{S}\right)^{\sigma}\right\}^{(1 / \sigma)-1}\left(H_{t}^{S}\right)^{\sigma-1} \\
& \frac{\partial F\left(A_{t}, K_{t}, H_{t}^{S}, H_{t}^{T}\right)}{\partial H_{t}^{T}}= \\
& A_{t} \mu\left\{\mu\left[K_{t}^{\varphi}+\left(H_{t}^{T}\right)^{\varphi}\right]^{\sigma / \varphi}+\left(H_{t}^{S}\right)^{\sigma}\right\}^{(1 / \sigma)-1}\left[K_{t}^{\varphi}+\left(H_{t}^{T}\right)^{\varphi}\right]^{(\sigma / \varphi)-1}\left(H_{t}^{T}\right)^{\varphi-1}
\end{aligned}
$$

habiendo definido $\Gamma$ en (47) y tomando (48) y (49), se obtiene (29).

\section{Anexo 7}

Cuadro 3: Factores de Producción (millones de ptas. constantes de 1993) (Base 1986).

\begin{tabular}{ccccc}
\hline Sectores & $\mathrm{K}$ & $\mathrm{Hs}$ & $\mathrm{Ht}$ & $\mathrm{Ht} / \mathrm{Hs}$ \\
\hline Agricultura & $2.408 \mathrm{E}+11$ & $1.531 \mathrm{E}+04$ & $5.857 \mathrm{E}+02$ & 0.038 \\
Construcció & $5.019 \mathrm{E}+10$ & $2.715 \mathrm{E}+04$ & $1.535 \mathrm{E}+03$ & 0.057 \\
$\mathrm{n}$ & & & & \\
Energía & $3.973 \mathrm{E}+11$ & $4.177 \mathrm{E}+03$ & $4.088 \mathrm{E}+03$ & 0.979 \\
Industria & $6.844 \mathrm{E}+11$ & $7.650 \mathrm{E}+04$ & $9.922 \mathrm{E}+03$ & 0.130 \\
\hline Servicios & $4.122 \mathrm{E}+12$ & $1.877 \mathrm{E}+05$ & $2.308 \mathrm{E}+04$ & 0.123 \\
\hline
\end{tabular}

Fuente: Elaboración propia a partir de datos del BBVA y Bancaja (año 1993) 


\section{Anexo 8}

Cuadro 4. Correlaciones. Sector Agrícola

\begin{tabular}{ccccc}
\hline & VAB & Hs & Ht & K \\
\hline VAB & 1,000 & 0,870 & $-0,064$ & 0,816 \\
Hs & 0,870 & 1,000 & $-0,126$ & 0,900 \\
Ht & $-0,064$ & $-0,126$ & 1,000 & $-0,163$ \\
\hline K & 0,816 & 0,900 & $-0,163$ & 1,000 \\
\hline
\end{tabular}

Cuadro 5. Correlaciones. Sector de la Construcción

\begin{tabular}{ccccc}
\hline & VAB & Hs & Ht & K \\
\hline VAB & 1,000 & 0,982 & 0,269 & 0,957 \\
Hs & 0,982 & 1,000 & 0,346 & 0,974 \\
Ht & 0,269 & 0,346 & 1,000 & 0,468 \\
\hline K & 0,957 & 0,974 & 0,468 & 1,000 \\
\hline
\end{tabular}

Cuadro 6. Correlaciones. Sector de la Energía.

\begin{tabular}{lllll}
\hline & VAB & Hs & Ht & K \\
\hline VAB & 1,000 & 0,855 & 0,400 & 0,972 \\
Hs & 0,855 & 1,000 & 0,445 & 0,784 \\
Ht & 0,400 & 0,445 & 1,000 & 0,235 \\
\hline K & 0,972 & 0,784 & 0,235 & 1,000 \\
\hline
\end{tabular}

Cuadro 7. Correlaciones. Sector de la Industria.

\begin{tabular}{ccccc}
\hline & VAB & Hs & Ht & K \\
\hline VAB & 1,000 & 0,996 & 0,821 & 0,984 \\
Hs & 0,996 & 1,000 & 0,804 & 0,968 \\
Ht & 0,821 & 0,804 & 1,000 & 0,819 \\
\hline K & 0,984 & 0,968 & 0,819 & 1,000 \\
\hline
\end{tabular}

Cuadro 8. Correlaciones. Sector Servicios.

\begin{tabular}{lllll}
\hline & VAB & Hs & Ht & K \\
\hline VAB & 1,000 & 0,992 & 0,856 & 0,945 \\
$\mathrm{Hs}$ & 0,992 & 1,000 & 0,863 & 0,955 \\
$\mathrm{Ht}$ & 0,856 & 0,863 & 1,000 & 0,952 \\
\hline $\mathrm{K}$ & 0,945 & 0,955 & 0,952 & 1,000 \\
\hline
\end{tabular}




\section{Anexo 9}

Utilizamos el método de mínimos cuadrados no lineales para estimar la función de producción (22). Dada la función $y_{i}=h\left(x_{i}, \beta\right)+\varepsilon_{i}$, el procedimiento de mínimos cuadrados no lineales, consiste en determinar el valor del vector de parámetros $\beta$ que minimice:

$$
S(\beta)=\frac{1}{2} \sum_{i=1}^{n}\left(\varepsilon_{i}\right)^{2}=\frac{1}{2} \sum_{i=1}^{n}\left(y_{i}-h\left(x_{i}, \beta\right)\right)^{2}
$$

Aplicando el algoritmo de Marquardt, se calcula la derivada con respecto a cada parámetro, se hace un pequeño cambio en los parámetros y evaluamos el cambio (cuando es necesario se aplica el método de Ridge Regression para resolver problemas de colinealidad).

El proceso de estimación parte de los siguientes valores iniciales: $A=100, \mu=1, \rho=0,25$ y $\sigma=1$. obtenidos.

Los Cuadros 9, 10, 11, 12 y 13 muestran los resultados

Cuadro 9. Estimación de parámetros. Agricultura.

\begin{tabular}{cccc}
\hline & Parámetros & $\begin{array}{c}\text { Error } \\
\text { Estándar }\end{array}$ & P-valor \\
\hline $\mathbf{A}$ & 77,180 & 13,3 & 0,0001 \\
$\mu$ & $6,70 \mathrm{E}-05$ & $1,82 \mathrm{E}-05$ & 0,0025 \\
$\mathbf{\Psi}$ & 0,273 & 0,211 & 0,0460 \\
$\mathbf{S}$ & 2,017 & 0,84 & 0,0306 \\
\hline $\mathbf{R}^{2}$ & & 0,721 & \\
\hline
\end{tabular}

Cuadro 10: Estimación de parámetros Construcción.

\begin{tabular}{cccc}
\hline & Parámetros & $\begin{array}{c}\text { Error } \\
\text { Estándar }\end{array}$ & P-valor \\
\hline $\mathbf{A}$ & $2,41 \mathrm{E}-05$ & $6,30 \mathrm{E}-06$ & 0,0024 \\
$\mu$ & 1,403 & 0,627 & 0,0352 \\
$\mathbf{\Psi}$ & 0,609 & 0,254 & 0,0306 \\
$\mathbf{S}$ & 0,070 & 0,019 & 0,0026 \\
\hline $\mathbf{R}^{2}$ & & 0,742 & \\
\hline
\end{tabular}


Cuadro 11. Estimación de parámetros. Energía.

\begin{tabular}{cccc}
\hline & Parámetros & $\begin{array}{c}\text { Error } \\
\text { Estándar }\end{array}$ & P-valor \\
\hline $\mathrm{A}$ & $6,24 \mathrm{E}-05$ & $3,03 \mathrm{E}-05$ & 0,0587 \\
$\mu$ & 2,062 & 0,250 & 0,0000 \\
$\Psi$ & 0,330 & 0,176 & 0,0817 \\
$\mathrm{~S}$ & 0,110 & 0,050 & 0,0511 \\
\hline $\mathbf{R}^{2}$ & & 0,698 & \\
\hline
\end{tabular}

Cuadro 12. Estimación de parámetros. Industria.

\begin{tabular}{cccc}
\hline & Parámetros & $\begin{array}{c}\text { Error } \\
\text { Estándar }\end{array}$ & P-valor \\
\hline $\mathbf{A}$ & $1,38 \mathrm{E}-05$ & $3,60 \mathrm{E}-06$ & 0,0016 \\
$\boldsymbol{\mu}$ & 1,340 & 0,248 & 0,0001 \\
$\mathbf{\Psi}$ & 0,130 & 0,031 & 0,0011 \\
$\mathbf{S}$ & 0,066 & 0,003 & 0,0000 \\
\hline $\mathbf{R}^{2}$ & \multicolumn{3}{c}{0,821} \\
\hline
\end{tabular}

Cuadro 13. Estimación de parámetros. Servicios.

\begin{tabular}{cccc}
\hline & Parámetros & $\begin{array}{c}\text { Error } \\
\text { Estándar }\end{array}$ & P-valor \\
\hline $\mathbf{A}$ & 87,804 & 2,022 & 0,0000 \\
$\mu$ & $1,10 \mathrm{E}-04$ & 3,753 & 0,0003 \\
$\mathbf{\Psi}$ & 1,359 & 0,256 & 0,0001 \\
$\mathbf{S}$ & 1,576 & 0,177 & 0,0000 \\
\hline $\mathbf{R}^{2}$ & & 0,765 & \\
\hline
\end{tabular}

De los cuadros se desprende que el factor tecnológico es mayor en el sector servicios que en el resto de sectores.

Como puede observarse $\varphi>\sigma$ en los sectores construcción, energía e industria. Por tanto, y de acuerdo con la Proposición 1, en estos sectores un aumento del capital físico conducirá a un incremento relativo de la productividad marginal de la educación con respecto a la formación en el puesto de trabajo. Por el contrario en la agricultura y servicios encontramos que $\varphi<\sigma$, de manera que el aumento del nivel de capital físico provocaría una disminución relativa de la productividad 
marginal de la educación con respecto a la formación en el puesto de trabajo. Conocido (27) y (28), y a partir de los valores de $\varphi$ y $\sigma$ dados en los Cuadros 9, 10, 11, 12 y 13, podemos obtener las correspondientes elasticidades de sustitución:

Tabla 14: Elasticidades de Sustitución.

\begin{tabular}{cccrcc}
\hline & Agricultura & Construcción & Energía & Industria & Servicios \\
\hline $\boldsymbol{E}_{H^{S}, \boldsymbol{H}^{T}}$ & $-0,9830$ & 1,0750 & 1,1240 & 1,0710 & $-1,7360$ \\
$\boldsymbol{\varepsilon} \boldsymbol{H}^{S}, \boldsymbol{K}$ & $-0,9830$ & 1,0750 & 1,1240 & 1,0710 & $-1,7360$ \\
\hline $\boldsymbol{\varepsilon}_{H^{T}}, \boldsymbol{K}$ & 1,3750 & 2,5570 & 1,4920 & 1,1490 & $-2,7850$ \\
\hline
\end{tabular}

De acuerdo con el Cuadro 14, tanto en el sector agrícola como en el sector servicios, la elasticidad de sustitución entre la educación en la escuela y la formación en el trabajo es negativa, lo que implica que existe un alto grado sustituibilidad entre estas dos formas de capital humano. En el resto de los sectores esta elasticidad resulta positiva, lo que nos indica que estas dos formas de capital humano son complementarias, siendo este grado de complementariedad mayor en el sector energético. Podemos observar una situación idéntica con relación a la elasticidad de sustitución entre capital físico y educación en la escuela.

Por otro lado, la elasticidad de sustitución entre capital físico y formación sólo resulta negativa en el sector servicios. Además, como puede observarse para los sectores construcción, energía e industria, el grado de complementariedad entre capital físico y formación en el trabajo es mayor que el correspondiente al capital físico y a la educación en la escuela. Por último es importante señalar que en el sector servicios, que por otro lado es el que presenta los mayores niveles de capital físico, todas las elasticidades presentan valores negativos, indicando que todas las formas de capital son sustitutivas entre sí. 